\title{
Experimental Study of a Low-Pressure Glow Discharge in Air in Large-Diameter Discharge Tubes: I. Conditions for the Normal Regime of a Glow Discharge
}

\author{
V. A. Lisovskiy* and S. D. Yakovin** \\ *Kharkov National University, pl. Svobody 4, Kharkov, 61077 Ukraine \\ **Scientific Physics and Technology Center, ul. Novgorodskaya 1, Kharkov, 61145 Ukraine \\ Received March 16, 2000; in final form, June 22, 2000
}

\begin{abstract}
The initiation and characteristics of a low-pressure glow discharge in air in large-diameter discharge tubes are studied. A deviation from the Paschen law is observed: the breakdown curves $U_{d c}(p L)$ shift toward the higher values of $U_{d c}$ and $p L$ as the interelectrode distance $L$ increases. It is shown that the normal regime of a glow discharge is accompanied by gas ionization in the anode sheath. This takes place only for $p L$ values lying to the right of the inflection point in the breakdown curve. The cathode-sheath characteristics in the normal and abnormal regimes of an air discharge for a duralumin cathode are determined. The axial profiles of the ion density, electron temperature, and plasma potential, as well as the anode voltage drop, are measured at various air pressures. () 2000 MAIK “Nauka/Interperiodica”.
\end{abstract}

\section{INTRODUCTION}

DC glow discharges are widely used to produce thin polymer and oxide films, clean material surfaces, and pump gas-discharge lasers. Glow discharges are also used in plasma display panels and gas-discharge switches, which are key elements in radio-electronic and electrotechnical devices. Therefore, studies of the breakdown conditions, discharge regimes, and structure of a glow discharge are of considerable interest.

As is known [1-7], dc glow discharges can occur in both normal and abnormal regimes. In the abnormal regime, the entire cathode is occupied by the discharge. In this case, the increase in the discharge current $I_{d c}$ is accompanied by an increase in both the voltage drop $U_{c}$ across the cathode sheath and the discharge voltage $U_{d c}$, as well as a decrease in the cathode sheath length $d_{c}$. Such behavior of the cathode voltage drop is observed for $U_{c}$ values higher than the so-called normal cathode voltage drop, which depends on the type of gas and cathode material. The normal regime of a glow discharge is characterized by the conditions $U_{c}=U_{n}$ and $p d_{c}=p d_{n}$. In this regime, only part of the cathode surface may be occupied by the discharge. As the discharge current decreases, the cathode voltage drop $U_{c}$ and the cathode-sheath length $d_{c}$ remain constant, whereas the cathode area $S$ occupied by the discharge decreases, so that the current density $j=I_{d c} / S$ does not change. In $[8,9]$, it is asserted that, at pressure values lying to the left of the minimum of the breakdown curve $p L<(p L)_{\min }$, where $p$ is the gas pressure and $L$ is the distance between the electrodes, a glow discharge can occur only in the abnormal regime, whereas the normal regime is observed for $p L \geq(p L)_{\min }$.

Experiments [1, 2, 10-13] were largely conducted in long $(L \sim 50 \mathrm{~cm})$ and narrow $(\sim 2-3 \mathrm{~cm}$ in diameter) discharge tubes. However, technological plasma devices often use discharge chambers in which the cathode diameter is much larger than the distance between the cathode and anode (below, such discharge chambers will be referred to as large-diameter tubes). Hence, it is of interest to clarify the properties of a glow discharge in large-diameter discharge tubes.

In this study, we measured the breakdown curves and $I-V$ characteristics of a glow discharge in air in large-diameter discharge tubes. The axial profiles of the plasma density, electron temperature, and plasma potential, as well as the cathode and anode voltage drops, were measured using the probe technique. It is shown that the normal discharge regime occurs at pressure values lying to the right of the inflection point in the breakdown curve. The normal regime of a discharge in air is accompanied by ionization in the anode sheath; in the absence of such ionization, the abnormal regime of a glow discharge takes place.

\section{EXPERIMENTAL TECHNIQUES}

Experiments were carried out at air pressures of $p=$ $10^{-2}-10$ torr, in the range of dc voltages $U_{d c} \leq 1000 \mathrm{~V}$ and discharge currents $I_{d c} \leq 100 \mathrm{~mA}$. A duralumin cathode was at the potential of a dc voltage source. A $1.5-\mathrm{k} \Omega$ resistor was connected in series to the discharge circuit between the cathode and the dc voltage source. A stainless-steel anode was grounded. 
Fragments of a quartz tube $100 \mathrm{~mm}$ in diameter were inserted and sealed between the cathode and anode. The use of a set of such quartz tubes of different lengths allowed us to discretely vary the distance between the cathode and anode within the range $L=$ 11-54 $\mathrm{mm}$. The outer cathode and anode diameters were equal to $160 \mathrm{~mm}$; i.e., they were larger than the inner discharge-tube diameter. Such a design of the discharge chamber allowed us to create a highly uniform electric field in a vacuum (in the absence of a discharge), thereby eliminating the edge effects during gas breakdown and measurements of the breakdown curves of a glow discharge.

The plasma parameters (electron temperature $T_{e}$, plasma density $n_{i}$, and plasma potential $\varphi_{p l}$ ) were measured with the help of single cylindrical nichrome probes $5 \mathrm{~mm}$ in length and $0.18 \mathrm{~mm}$ in diameter. In the pressure range under investigation, all three (collisionless, transition, and collisional) regimes of probe operation can occur: at different pressures, the gas ions pass through the probe sheath either without colliding with gas molecules (at low pressures of $p \leq 0.05$ torr) or colliding several times (at intermediate pressures) or frequently (at $p>1$ torr) with neutrals. For this reason, the plasma density $n_{i}$ was determined from the ion branch of the probe $I-V$ characteristic and from the measured values of the electron temperature $T_{e}$ using the procedure described in $[14,15]$. The plasma potential $\varphi_{p l}$ was derived from the zero of the second derivative of the probe current with respect to the probe voltage and also from measured values of the probe floating potential $\varphi_{f}$ and the electron temperature $T_{e}$ using the formula $\varphi_{p l}=$ $\varphi_{f}+C T_{e}$, where $C$ is a constant depending on the type of gas [6].

The electron temperature $T_{e}$ was determined from the linear regions in the probe $I-V$ characteristic and in the second derivative of the probe current with respect to the probe voltage (plotted on the semilogarithmic scale). The $T_{e}$ values determined by these two methods differed by no more than 10-20\%. When measuring the second derivative of the probe current with respect to the voltage, $d^{2} I_{p r} / d U_{p r}^{2}$, we used the second-harmonic method: the probe current was modulated with a lowfrequency $\left(f_{l f} \sim 1-3 \mathrm{kHz}\right)$ voltage, and a signal at a frequency of $2 f_{l f}$ was detected. Since, in the pressure range under study, electrons collide with gas molecules near the probe, the method of determining $T_{e}$ from the slope of the $I-V$ characteristic can give an overestimated value of the electron temperature. However, as was shown in $[6,16]$, the electron temperature in the collisional regime can be determined from the slope of the linear region in the $I-V$ characteristic (plotted on the semilogarithmic scale) near the probe floating potential [17], rather than near the plasma potential. In our experiments, the method for determining $T_{e}$ proposed in [17] was used for the entire pressure range under study. The electron temperature determined in this way was usually 2-3 times lower than the $T_{e}$ determined from the slope of the linear region in the probe $I-V$ characteristic near the plasma potential.

\section{ANALYSIS OF EXPERIMENTAL RESULTS}

\subsection{Conditions for the Normal Regime of a Glow Discharge}

In this section, we present results from studies of the relation between the breakdown curves and the $I-V$ characteristic of a glow discharge. In particular, we determine the lowest pressure for which the effect of the normal current density can exist.
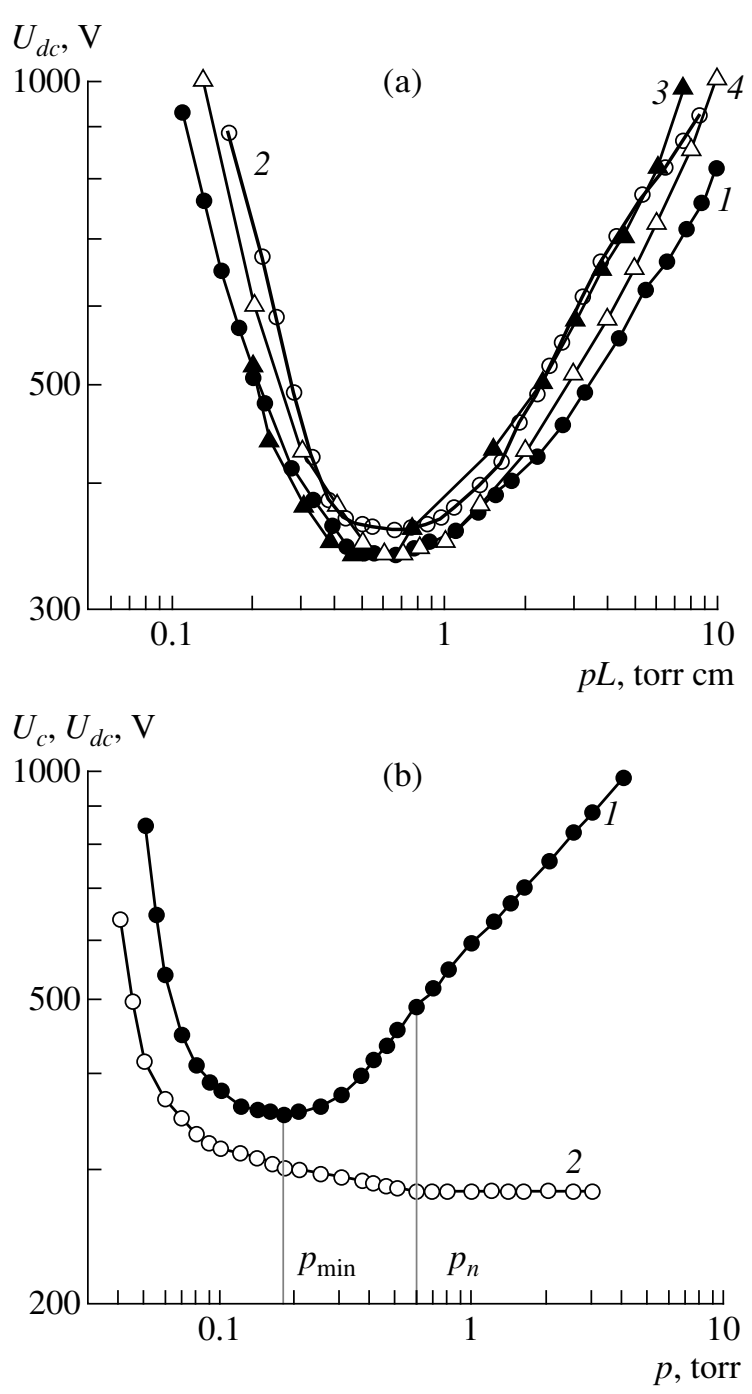

Fig. 1. (a) Breakdown curves of a glow discharge in air: (1) $L=11$, (2) $L=54 \mathrm{~mm}$, (3) experiment [19], and (4) experiment [16]; (b) (1) breakdown curve of a glow discharge and (2) dependence of the minimum value of the cathode voltage drop on the air pressure for $L=32 \mathrm{~mm}$. 


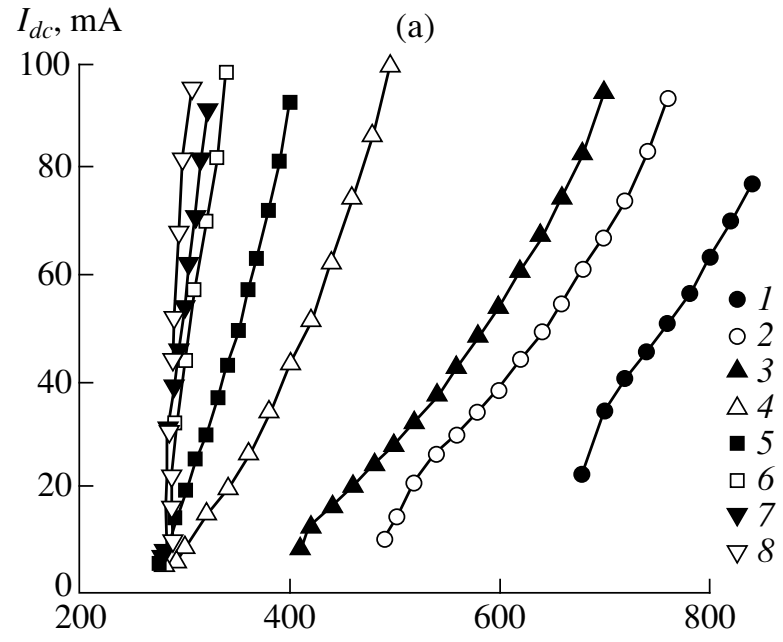

(b)

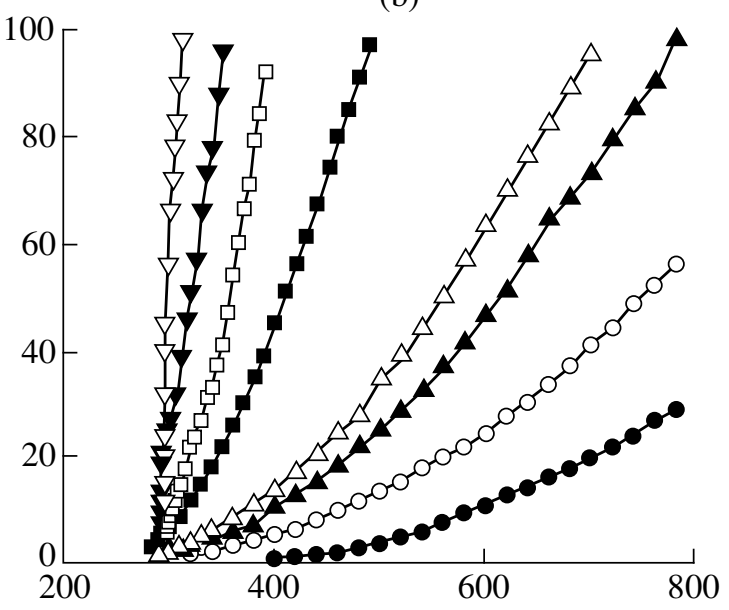

(c)

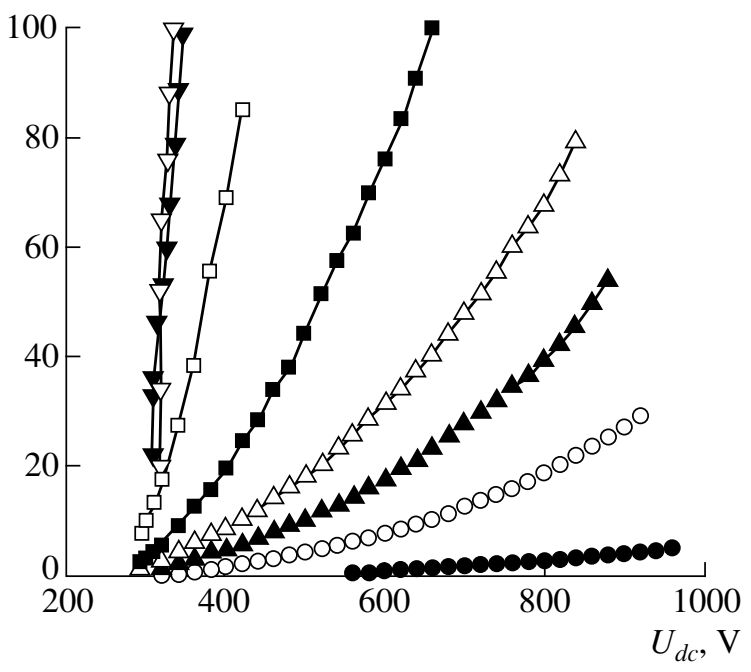

Fig. 2. $I-V$ characteristics of a glow discharge for (a) $L=$ $11 \mathrm{~mm}$ at $p=$ (1) 0.1, (2) 0.15 , (3) 0.2, (4) 0.4, (5) 0.6, (6) 1 , (7) 1.5 , and (8) 2 torr; (b) for $L=32 \mathrm{~mm}$ at $p=(1) 0.06$, (2) $0.1,(3) 0.15$, , (4) 0.2, , (5) 0.4, , (6) 0.6, , (7) 1 , and (8) 2 torr; and (c) for $L=54 \mathrm{~mm}$ at $p=(1) 0.04$, (2) 0.1 , (3) 0.15 , (4) $0.2,(5) 0.3,(6) 0.6,(7) 1.25$, and (8) 2 torr.
Figure 1 shows the breakdown curves of a glow discharge in air, $U_{d c}(p L)$, measured by us for different distances $L$ between the cathode and anode. It is seen from the figure that, as $L$ increases, the minimum of the breakdown curve shifts toward higher values of the product $p L$. For example, for $L=1.1 \mathrm{~cm}$, we have $p_{\min } \approx$ 0.55 torr and $(p L)_{\min } \approx 0.61$ torr $\mathrm{cm}$; for $L=5.4 \mathrm{~cm}$, we have $p_{\min } \approx 0.12$ torr and $(p L)_{\min } \approx 0.65$ torr $\mathrm{cm}$. At the same time, the breakdown voltage $U_{d c}$ increases over the entire range of $p L$ as the distance $L$ between the cathode and anode increases. Thus, for $p L=0.6$ torr $\mathrm{cm}$ (near the minimums of the breakdown curves), a glow discharge arises at $U_{d c}=334 \mathrm{~V}$ for $L=1.1 \mathrm{~cm}$ and $U_{d c}=$ $360 \mathrm{~V}$ for $L=5.4 \mathrm{~cm}$. The same dependence $U_{d c}(L)$ at $p L=$ const was observed for other $p L$ values. Hence, an increase in $L$ leads to the shift of the breakdown curves toward higher values of both $p L$ and the breakdown voltage $U_{d c}$. An increase in the breakdown voltage $U_{d c}(p L)$ was also indicated in $[18,19]$. Such a deviation from the Paschen law can be attributed to the fact that the loss of charge particles due to diffusion toward the side walls of the discharge tube increases as the distance between the cathode and anode increases. A theoretical description of this effect is given in [20,21].

Figure 1a also demonstrates the breakdown curves obtained by other authors $[22,23]$. Note that curves 3 and 4 in Fig. 1a were obtained by averaging many experimental breakdown curves for different values of the interelectrode distance and the discharge-tube diameter. It is seen from the figure that our breakdown curves agree satisfactorily with the results of $[22,23]$.

Now, we specify the values of $p L$ at which different structural components of a glow discharge are observed. At $p L<(p L)_{\min }$ and over the entire range of $U_{d c}$, the glow discharge consists of a cathode sheath and a negative glow. At low values of $U_{d c}$, the cathode sheath occupies almost the entire discharge gap and the negative glow is adjacent to the anode. As $U_{d c}$ increases, the length of the cathode sheath decreases, whereas almost the entire remaining part of the discharge gap becomes occupied by the negative glow. In this range of $p L$, the anode sheath is almost invisible, especially at higher values of the applied voltage $U_{d c}$.

At $p L \approx(p L)_{\min }$ and low values of $U_{d c}$, a Faraday dark space appears; however, at a higher $U_{d c}$, the Faraday dark space disappears and the glow discharge again consists of the cathode sheath and the negative glow.

For $(p L)_{\text {inf }}=e(p L)_{\min }$ (where $e$ is the base of the natural logarithm), the Townsend breakdown criterion predicts the occurrence of an inflection point in the breakdown curve of a glow discharge [6, 20,23]. Experimentally, such a point is sometimes absent in the breakdown curve. At $p L \geq(p L)_{\text {inf }}$, the glow discharge includes not only the cathode sheath, the negative glow, and the Faraday dark space, but also the anode sheath with the anode glow. 
At $p L>50$ torr $\mathrm{cm}$ (i.e., at $p L \gg(p L)_{\min }$ ), a positive column arises. No inflection points are observed on the breakdown curve in this case.

Figure 2 shows the $I-V$ characteristics of a glow discharge, $I_{d c}=f\left(U_{d c}\right)$, for different distances $L$ between the cathode and anode. It is seen from Fig. 2a that, for a short interelectrode distance $(L=1.1 \mathrm{~cm})$, the discharge current is relatively high $\left(I_{d c} \geq 5 \mathrm{~mA}\right)$ even at low gas pressures. At the minimum applied voltage $U_{d c}$, the discharge only partially occupies the cathode and anode surfaces. As $U_{d c}$ increases, the discharge current $I_{d c}$ increases and the electrode area occupied by the discharge increases rapidly until it occupies the entire electrode surface. At low pressures $\left(p L \leq(p L)_{\text {inf }}\right)$, the effect of the normal current density is not observed because the increase in the discharge current is always accompanied by an increase in the discharge voltage. For $L=1.1 \mathrm{~cm}$, the effect of the normal current density is observed at $p \approx 1.5$ torr, i.e., $(p L)_{n} \approx 1.65$ torr $\mathrm{cm}$. Since, for this value of $L$, the minimum of the breakdown curve lies at $(p L)_{\min } \approx 0.61$ torr $\mathrm{cm}$, we obtain that $(p L)_{n} \approx 2.71(p L)_{\min }$.

If at $p L \geq(p L)_{\text {inf }}$, the discharge voltage is reduced after breakdown, then the discharge is first observed to occur in the abnormal regime and to uniformly occupy the entire area of the electrodes. Then, the discharge arrives at the normal regime, in which case it is located near the dielectric side wall of the discharge tube, whereas the discharge at the center of the cathode is quenched. As the current $I_{d c}$ decreases, the discharge in the normal regime first takes the shape of a torus. Then, a break in the torus appears and the discharge takes the shape of a toroidal segment. After this, the discharge is quenched.

The $I-V$ characteristics for long interelectrode distances $L$ are shown in Figs. 2b and 2c. At low values of the gas pressure and the voltage $U_{d c}$, the discharge current is low and, before quenching, the discharge occupies the entire cross section of the discharge tube. For $L=3.2 \mathrm{~cm}$, the effect of the normal current density appears at $p \approx 0.6$ torr, i.e., $(p L)_{n} \approx 3.2(p L)_{\min }$. As for $L=$ $1.1 \mathrm{~cm}$, for this distance, the normal discharge regime is observed at a gas pressure such that an anode glow (clearly visible and uniform over the entire anode surface) arises near the anode. As the pressure increases, the anode glow first appears near the wall of the discharge tube; then, the glow expands over the anode surface into the central region. When the anode glow at the minimum discharge voltage uniformly covers the entire anode surface, the effect of the normal current density appears.

This result is very interesting. Before, it was commonly accepted that the processes occurring in the cathode sheath are only important for the effect of the normal current density; i.e., it is necessary that the voltage drop across the cathode sheath become equal to the normal cathode voltage drop $U_{c}=U_{n}$ and the cathode- sheath length be equal to the normal length $d_{c}=d_{n}$. At the same time, little attention was given to the shape of the discharge and the processes occurring in the entire discharge gap, rather than only in the cathode sheath.

The anode glow appears when the voltage drop across the anode sheath becomes close to the ionization potential for neutral gas particles. Hence, it may be concluded that the voltage drop across the anode sheath becomes comparable with the ionization potential when the effect of the normal current density takes place in large-diameter discharge tubes; i.e., the gas molecules in the anode sheath are ionized by electron impact. A comparison of $(p L)_{n}$ values for $L=1.1$ and $3.2 \mathrm{~cm}$ allows us to conclude that $(p L)_{n} \approx(p L)_{\text {inf }}$; i.e., the normal regime of a glow discharge can occur only within the pressure range to the right of the inflection point in the breakdown curve (at $p L>(p L)_{\text {inf }}$ ).

Figure $2 \mathrm{c}$ shows the $I-V$ characteristics of a discharge at $L=5.4 \mathrm{~cm}$. Qualitatively, they are similar to the characteristics shown in Fig. 2b, but there are some differences. For $L=5.4 \mathrm{~cm}$, the anode glow appears at $p \approx 0.3$ torr, i.e., $p L \approx 1.62$ torr $\mathrm{cm}\left(p L \approx 2.5(p L)_{\min }\right)$. The normal discharge regime appears at an air pressure of $p \approx 1$ torr (for $p L \approx 8.3(p L)_{\text {min }}$ ); i.e., there is a substantial difference in $p L$ between the appearance of the anode glow and the effect of the normal current density. However, as is the case for shorter distances $L$, the normal regime takes place only after a clearly visible anode glow appears near the anode surface.

We also carried out a series of experiments in argon and nitrogen and obtained the following results. For argon at $L=1.1 \mathrm{~cm}$, the anode glow and the effect of the normal current density appear at $p L \approx(p L)_{n} \approx$ $2.68(p L)_{\min }$; for $L=2.2 \mathrm{~cm}$, the anode glow is observed at $p L \approx 2.72(p L)_{\min }$ and the normal discharge regime appears at $(p L)_{n} \approx 2.8(p L)_{\min }$. For nitrogen at $L=$ $1.1 \mathrm{~cm}$, the anode glow and the effect of the normal current density are observed starting from $p L \approx(p L)_{n} \approx$ $2.73(p L)_{\min }$. For $L=2.2 \mathrm{~cm}$, we have $p L \approx 2.7(p L)_{\min }$ for the appearance of the anode glow and $(p L)_{n} \approx$ $2.75(p L)_{\min }$ for the appearance of the normal discharge regime. For $L=3.3 \mathrm{~cm}$, the anode glow is observed starting from $p L \approx 2.69(p L)_{\min }$ and the effect of the normal current density appears at $(p L)_{n} \approx 2.85(p L)_{\text {min. }}$. This allows us to conclude that, over the entire range of the interelectrode distances $L$ under investigation, the anode glow in different gases appears at $p L \approx(p L)_{\text {inf }}$, i.e., at the inflection point in the breakdown curve of a glow discharge. With short interelectrode distances $L \leq 3 \mathrm{~cm}$, the normal discharge regime is observed at $(p L)_{n} \geq(p L)_{\text {inf }}$, whereas for large values of $L$, the effect of the normal current density exists only at $(p L)_{n}>$ $(p L)_{\text {inf. As }}$ As distance $L$ increases, the ratio $(p L)_{n} /(p L)_{\text {inf }}$ also increases. 


\subsection{Characteristics of the Cathode Sheath}

The processes occurring in the cathode sheath play a decisive role in sustaining a glow discharge. Therefore, it is not surprising that, in studying a glow discharge, much attention is frequently paid to the characteristics of the cathode sheath (see, e.g., [3, 7, 24-31]). We also measured such important characteristics as the cathode voltage drop $U_{c}$ (i.e., the voltage drop across the cathode sheath) and the cathode-sheath length $d_{c}$.

The voltage drop across the cathode sheath was determined by measuring the plasma potential $\varphi_{p l}$ with respect to the anode with the help of a cylindrical probe positioned parallel to the cathode surface at the visible boundary of the sheath. In this case, the cathode voltage drop $U_{c}$ is equal to the difference between the applied voltage $U_{d c}$ and the plasma potential; i.e., $U_{c}=U_{d c}-$ $\varphi_{p l}$. All of the probe measurements were made for the interelectrode distance $L=3.2 \mathrm{~cm}$.

Figure $1 \mathrm{~b}$ shows the dependence of a minimum cathode voltage drop on the gas pressure $p$. By a minimum cathode voltage drop, we mean the voltage drop across the cathode sheath before quenching the discharge. It is seen from the figure that, as the gas pressure increases, the minimum cathode voltage drop decreases, reaching the minimum value $U_{c}=U_{n}=$ $280 \pm 2 \mathrm{~V}$ at $p \sim 0.6$ torr. As the pressure increases further, this value remains almost unchanged and is equal to the normal cathode voltage drop for the air-duralumin cathode system. From the same figure, it also follows that the cathode voltage drop reaches the minimum value $U_{n}$ at $p L=(p L)_{n}$, i.e., when the effect of the normal current density takes place. For comparison, the

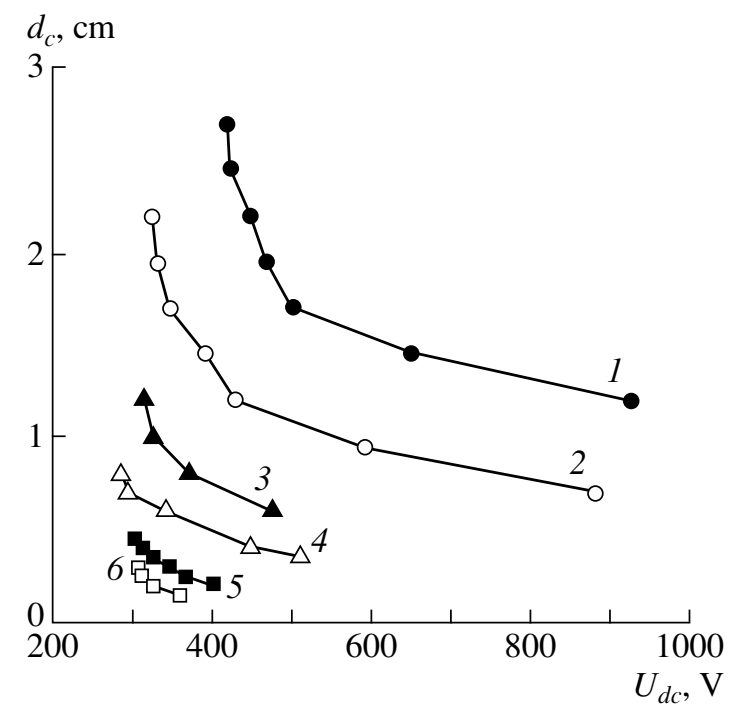

Fig. 3. The cathode-sheath length as a function of the constant voltage applied to the electrodes for $L=32 \mathrm{~mm}$ at $p=$ (1) 0.05 , (2) 0.1, (3) 0.2, (4) 0.3 , (5) 0.6 , and (6) 1 torr. breakdown curve of a glow discharge is also shown in Fig. 1b. It is easy to see that the cathode voltage drop becomes equal to its "normal" value at $p L>(p L)_{\text {inf }}$, rather than at $p L>(p L)_{\min }$ as was stated in $[8,9]$.

Figure 3 shows the dependence of the cathodesheath length $d_{c}$ on the applied voltage $U_{d c}$ for different air pressures. It follows from the figure that, at low pressures and low voltages $U_{d c}$, the cathode-sheath length is only slightly shorter than the interelectrode distance $L$, whereas at sufficiently high pressures, it covers only a small fraction of the discharge gap. The cathode-sheath length decreases rapidly with increasing applied voltage $U_{d c}$.

Figure $4 \mathrm{a}$ shows the cathode voltage drop $U_{c}$ for different values of the product $p d_{c}$. It is seen from the figure that the product of the normal sheath length and air pressure is $p d_{n} \approx 0.3$ torr $\mathrm{cm}$. All the experimental points fall reasonably well on one curve (which is analogous to the left branch of the Paschen curve).

Figure $4 \mathrm{~b}$ shows the ratio $j / p^{2}$ as a function of the cathode voltage drop $U_{c}$. It is seen from this figure that, first, the minimum (normal) value of this ratio is equal to $\left(j / p^{2}\right)_{n} \approx 0.21 \mathrm{~mA} / \mathrm{cm}^{2}$ torr ${ }^{2}$ and, second, all of the measured values of $j / p^{2}\left(U_{c}\right)$ also fall on one curve.

For comparison, we present the results obtained by other authors for air [1]: $U_{n}=229 \mathrm{~V}$ for an aluminum cathode and $U_{n}=269 \mathrm{~V}$ for an iron cathode, $\left(j / p^{2}\right)_{n} \approx$ $0.33 \mathrm{~mA} / \mathrm{cm}^{2}$ torr $^{2}$, and $p d_{n} \approx 0.25$ torr $\mathrm{cm}$. These characteristics of the normal regime of a glow discharge coincide reasonably well with our results.

\subsection{Axial Structure of a Glow Discharge}

Here, we consider the axial profiles of the plasma parameters (ion density, plasma potential, and electron temperature) for two fixed values of the air pressure: 0.1 torr (the left branch of the breakdown curve) and 0.6 torr (the right branch of the breakdown curve, when the effect of the normal current density takes place). Note that the $z$-coordinate in all of the axial profiles is measured from the anode; i.e., the anode is on the left (at $z=0$ ), and the cathode is on the right (at $z=3.2 \mathrm{~cm}$ ).

Figure 5 shows the axial profiles of the positive-ion density $n_{i}(z)$. For $p=0.1$ torr, the glow discharge consists of the cathode sheath, negative glow, and almost invisible anode sheath. From Fig. 5a, it is seen that, at low discharge voltages, the cathode sheath covers a significant fraction of the discharge gap and the cathodesheath length decreases rapidly with increasing $U_{d c}$. Near the anode, the ion density begins to fall rapidly starting from $z \approx 4-5 \mathrm{~mm}$.

For $p=0.6$ torr (Fig. 5b) and the minimum applied voltage $U_{d c}=300 \mathrm{~V}$, the normal regime takes place and the glow discharge consists of the cathode sheath (with length $d_{c} \leq 0.5 \mathrm{~cm}$ ), negative glow, Faraday dark space, and anode sheath with the anode glow. The length of 

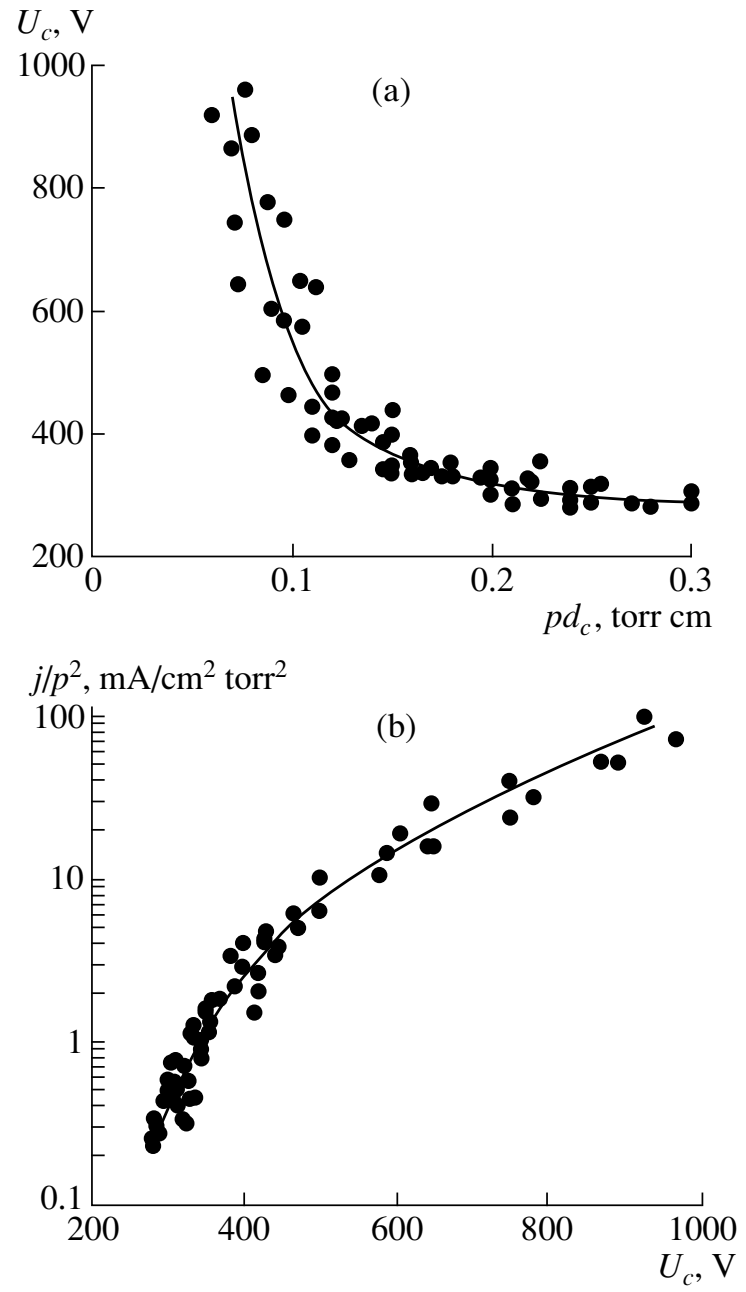

Fig. 4. (a) The cathode voltage drop as a function of the product $p d_{c}$ and (b) the ratio $j / p^{2}$ as a function of the cathode voltage drop for $L=32 \mathrm{~mm}$.

the anode glow is no more than $1-1.5 \mathrm{~mm}$, and the anode-sheath length is $d_{a} \approx 4 \mathrm{~mm}$. As the applied voltage $U_{d c}$ increases, the plasma density increases monotonically throughout the discharge gap and the negative glow expands toward the anode. First, the Faraday dark space disappears. When the boundary of the negative glow reaches the anode sheath, the anode glow decays and also disappears. The maximum plasma density is observed near the boundary of the cathode sheath in the negative glow of the discharge. At $p=0.1$ torr, the plasma density between the cathode and anode sheaths varies slightly (by no more than $30 \%$ for the range of voltages $U_{d c}$ under study), but at $p=0.6$ torr, the plasma density $n_{i}$ decreases from the cathode sheath to the anode sheath by a factor of 7-10.

Figure 6 shows the axial profiles of the plasma potential $\varphi_{p l}(z)$. At the air pressure $p=0.1$ torr, the plasma potential with respect to the anode in the negative glow was no more than $2 \mathrm{~V}$, while the remainder of

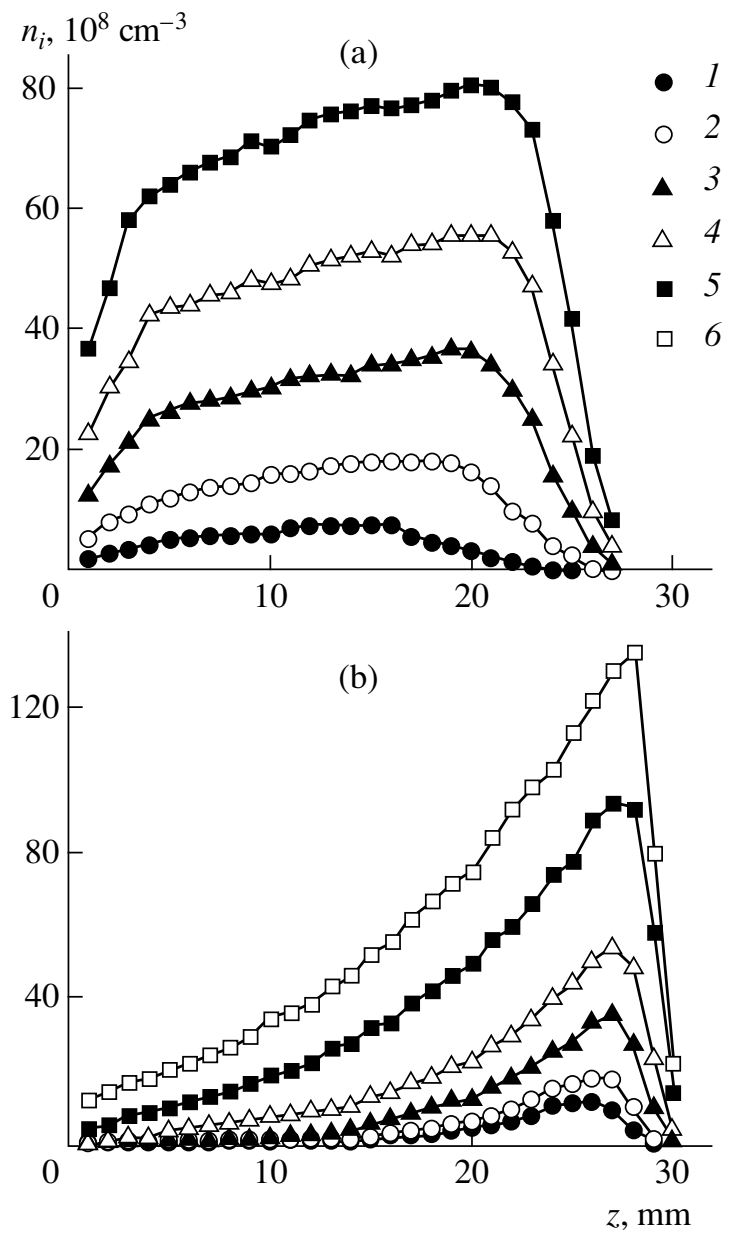

Fig. 5. Axial profiles of the plasma density in a glow discharge for $L=32 \mathrm{~mm}$ (a) at $p=0.1$ torr and $U_{d c}=(1) 350$, (2) 400 , (3) 500, (4) 600, and (5) $700 \mathrm{~V}$ and (b) at $p=0.6$ torr and $U_{d c}=$ (1) 300, (2) 325, (3) 350, (4) 375, (5) 400, and (6) $425 \mathrm{~V}$.

the voltage drop fell at the cathode sheath. The plasma potential in the negative glow slightly increased with applied voltage. In this case, the field in the anode sheath was nearly equal to the field in the negative glow. At $p=0.6$ torr and low values of $U_{d c}$, a significant voltage drop ( $10-12 \mathrm{~V})$ was observed in the anode sheath. In this case in the Faraday dark space together with the negative glow, the voltage drop was no more than 5-8 V. As the voltage $U_{d c}$ increased, the plasma potential with respect to the anode decreased to low values $(\sim 2-3 \mathrm{~V})$ almost throughout the entire discharge gap (except for the cathode sheath); at the same time, a nearly constant plasma potential was observed in the negative glow (i.e., the electric field in the negative glow almost vanished).

Figure 7 shows the axial profiles of the electron temperature $T_{e}(z)$. At $p=0.1$ torr (Fig. 7a) and low voltages $U_{d c}$, the electron temperature near the anode reaches the value $T_{e} \approx 2 \mathrm{eV}$ and decreases with distance from the 

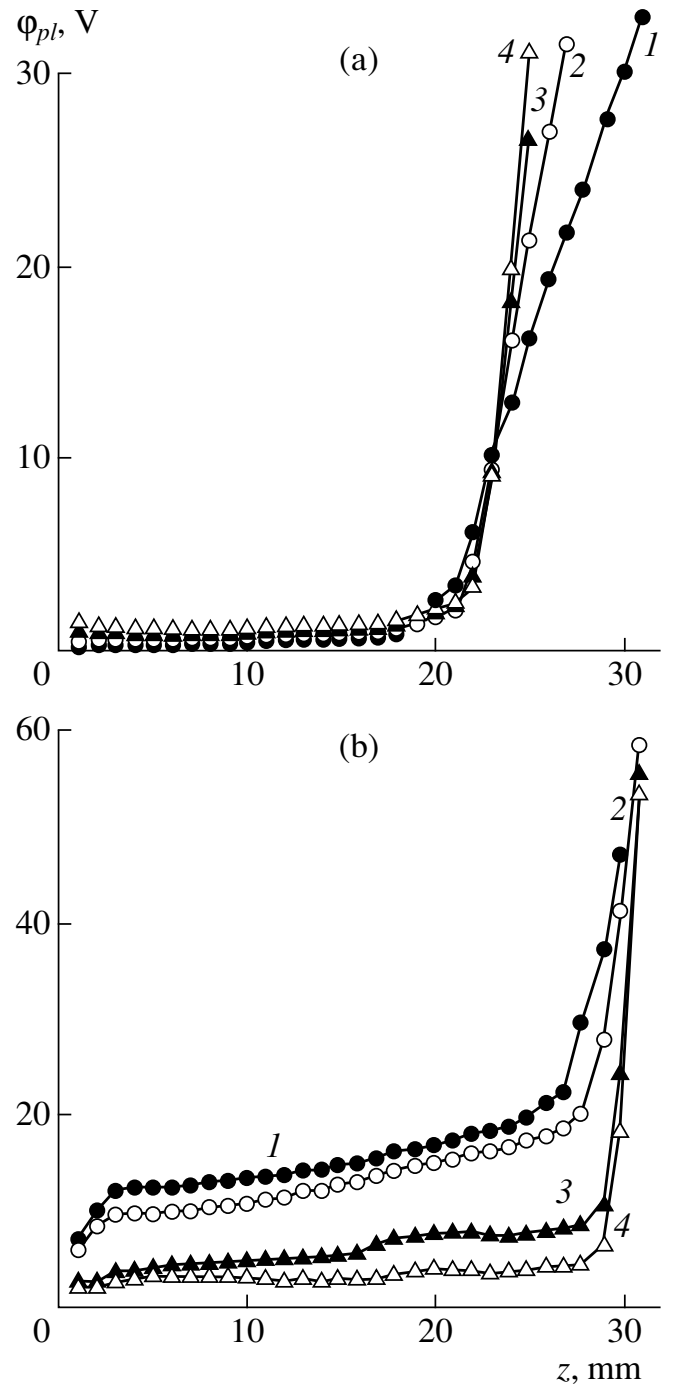

Fig. 6. Axial profiles of the plasma potential in a glow discharge for $L=32 \mathrm{~mm}$ (a) at $p=0.1$ torr and $U_{d c}=(1) 400$, (2) 500, (3) 600, and (4) $700 \mathrm{~V}$ and (b) at $p=0.6$ torr and $U_{d c}=$ (1) 300, (2) 350, (3) 400, and (4) $425 \mathrm{~V}$.

anode. Near the boundary of the cathode sheath and in the sheath itself, $T_{e}$ rapidly increases. At high discharge voltages, the electron temperature is $T_{e}<1 \mathrm{eV}$ almost throughout the entire discharge gap (except for the cathode sheath) and it somewhat increases near the anode.

At $p=0.6$ torr (Fig. 7b) and low discharge voltages, the electron temperature near the anode reaches the value $T_{e} \approx 6 \mathrm{eV}$, whereas at the boundary of the anode sheath, it is $T_{e} \approx 2-3 \mathrm{eV}$. In the Faraday dark space and negative glow, the electron temperature is $T_{e}<2 \mathrm{eV}$. As the voltage $U_{d c}$ increases, the anode glow disappears and the electron temperature in the anode sheath decreases sharply. At high values of $U_{d c}$, the electron temperature in the anode sheath and negative glow var-
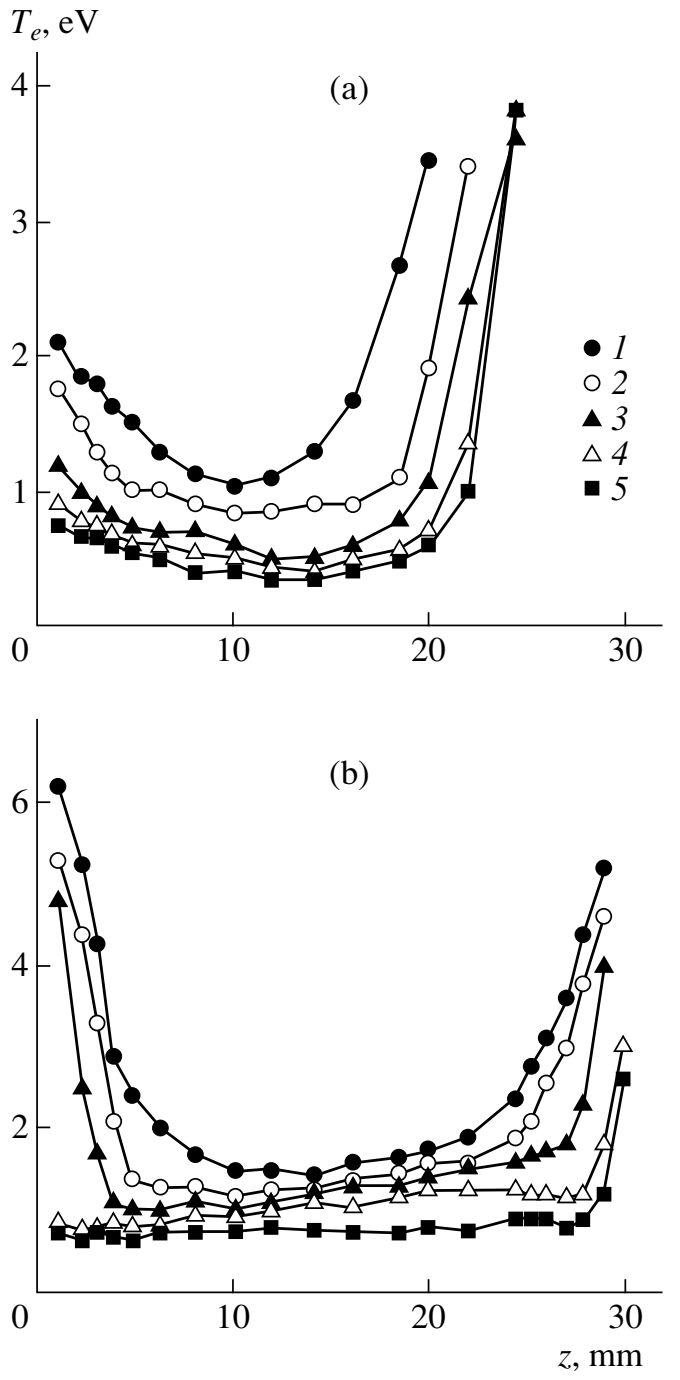

Fig. 7. Axial profiles of the electron temperature in a glow discharge for $L=32 \mathrm{~mm}$ (a) at $p=0.1$ torr and $U_{d c}=(1) 350$, (2) 400 , (3) 500, (4) 600, and (5) $700 \mathrm{~V}$ and (b) at $p=0.6$ torr and $U_{d c}=(1) 300$, (2) 325, (3) 350, (4) 400, and (5) $425 \mathrm{~V}$.

ies slightly with distance from the anode and is about $T_{e} \leq 1 \mathrm{eV}$.

We note that the sharp increase in the electron temperature near the boundary of the cathode sheath was also observed experimentally in [2, 32-35] and was predicted theoretically in $[26,36,37]$. This is associated primarily with an increase in the electric field and, as a result, with heating of electrons from the lowenergy part of the electron Maxwell distribution. Let us estimate how rapidly the electrons that acquire a higher temperature $T_{e}$ due to heating near the boundary of the cathode sheath will lose energy as they move deep into the negative glow. The mean free path of electrons in air

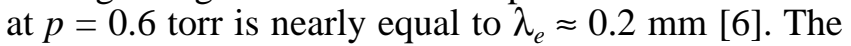
energy-relaxation length is equal to $\Lambda_{u} \approx 0.8 \lambda_{e} / \sqrt{\delta}$ [6], 
where $\delta$ is the fraction of energy lost by an electron in a collision with a gas molecule. For a collision between an electron with energy $\varepsilon_{e} \approx 4 \mathrm{eV}$ and a nitrogen molecule, we take $\delta \approx 0.05$ [38]. Then, we obtain $\Lambda_{u} \approx$ $1.8 \mathrm{~mm}$, which agrees with the results of our measurements. Electrons with energies $\varepsilon_{e} \geq 6.2 \mathrm{eV}$ can excite the electronic levels of nitrogen molecules [6], which increases $\delta$ and, in turn, decreases the energy-relaxation length for electrons.

Now, we consider the reason why the electron temperature increases near the anode. As will be shown below, the anode voltage drop at low air pressures is small $\left(U_{a} \approx 1-2 \mathrm{~V}\right)$; consequently, near the anode surface, the ratio of the electric field strength to the gas pressure at $p=0.1$ torr is no more than $E / p=30 \mathrm{~V} /(\mathrm{cm}$ torr). For this value of $E / p$, the electron temperature near the anode can reach a value of $T_{e}=2-3 \mathrm{eV}$ [39] (which is seen in Fig. 7a). For the air pressure $p=0.6$ torr and voltage $U_{d c}=300 \mathrm{~V}$, we obtain $E / p=200 \mathrm{~V} /(\mathrm{cm}$ torr) near the anode surface, which corresponds to an electron temperature of $T_{e}=6-8 \mathrm{eV}[39,40]$ and agrees with the results of our experiments. At a higher discharge voltage $U_{d c}$, when the fast electrons accelerated in the cathode sheath reach the anode and the anode glow disappears, the anode voltage drop sharply decreases, which results in a decrease in $E / p$ near the anode surface and a decrease in the electron temperature in the anode sheath. The increase in the electron temperature as the anode is approached was predicted theoretically in [11, 41-43] and observed experimentally in $[11,43]$.

\subsection{Anode Voltage Drop}

Figure 8 shows the measured voltage drop $U_{a}$ across the anode sheath. At low levels of air pressure, as the voltage $U_{d c}$ increases, the anode voltage drop slightly decreases, reaches its minimum, and then slightly increases; its value is $U_{a} \leq 2-3 \mathrm{~V}$. As the air pressure increases, the anode voltage drop increases (at a fixed value of $U_{d c}$ ). With the appearance of the anode glow, the voltage drop across the anode sheath sharply increases: $U_{a} \geq 10 \mathrm{~V}$. At the air pressure $p=0.6$ torr, the normal regime of a glow discharge is observed. In this case, the anode voltage drop is $U_{a} \geq 12 \mathrm{~V}$ and the electron temperature at the boundary of the anode sheath is $T_{e} \approx 3 \mathrm{eV}$; therefore, a certain fraction of the electrons moving toward the anode can acquire an energy that is sufficient to ionize gas molecules (for nitrogen molecules, the ionization energy is $U_{i}=15.6 \mathrm{eV}$; for oxygen molecules, it is $U_{i}=12.2 \mathrm{eV}$ ).

The anode voltage drop decreases as the voltage $U_{d c}$ increases. At $U_{d c}$ values such that the boundary of the negative glow approaches the anode sheath, the anode glow disappears and the anode voltage drop sharply decreases to $U_{a} \approx 3-5 \mathrm{~V}$ and remains almost unchanged as $U_{d c}$ further increases. The higher the air pressure, the

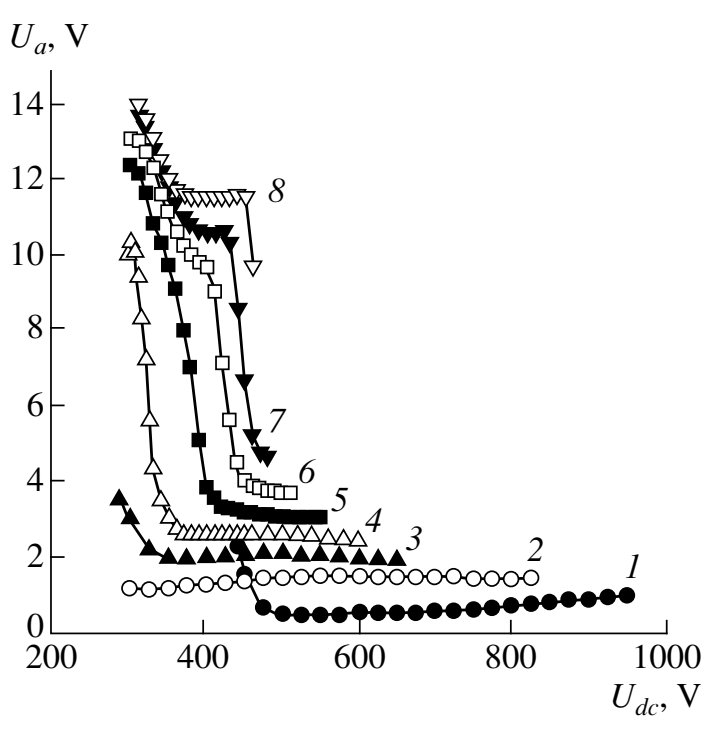

Fig. 8. Dependence of the anode voltage drop on the discharge voltage for $L=32 \mathrm{~mm}$ at air pressures $p=(1) 0.05$, (2) 0.2, , (3) 0.4 , (4) 0.5 , (5) 0.6, (6) 0.7, (7) 0.8 , and (8) 1 torr.

higher the voltage $U_{d c}$ at which the boundary of the negative glow approaches the anode sheath. Once the anode glow disappears, $U_{a}$ falls abruptly.

In a glow discharge, the anode acts as a collector of electrons that makes the electron current in the external circuit equal to the total discharge current. As is seen from Fig. 8, the value of the anode voltage drop depends strongly on the anode position in the discharge. If the anode is inside the negative glow or in the adjacent region of the Faraday dark space, then the anode voltage drop is small and increases slightly as the gas pressure or the discharge voltage $U_{d c}$ increases. In this region of the discharge gap, there is a significant flux of fast electrons emitted from the cathode surface and accelerated in the cathode sheath $[26,28]$. In addition, the plasma density is maximum in the negative glow (see Fig. 5 and also [1, 2]). Therefore, if the anode is inside the negative glow, then it collects a high diffusion electron current and a flow of fast electrons. These two electron flows can reach the anode surface even at relatively low values of the anode voltage drop (as is seen in Fig. 8). This is what usually takes place at sufficiently low gas pressure values lying near and to the left of the minimum of the breakdown curve of a glow discharge.

Another situation takes place when the anode lies sufficiently deep in the Faraday dark space (on the anode side of the Faraday dark space). In this region of glow discharge, the diffusion electron current is low (which is seen from Fig. 5). Most of the fast electrons accelerated in the cathode sheath cannot penetrate into this region of the discharge, because they lose a great deal of energy in inelastic and elastic collisions with gas molecules and are thermalized. In such a situation, an additional ionization in the anode sheath is required 
to make the electron current in the external circuit equal to the total discharge current. Hence, the anode voltage drop should be comparable with the ionization potential of gas molecules by electron impact. In this case, the anode glow is observed near the anode surface. As follows from the results of this study, this situation takes place at gas pressure values lying to the right of the inflection point of the breakdown curve of a glow discharge and moderate discharge voltages $U_{d c}$. As $U_{d c}$ increases, the boundary between the negative glow and the Faraday dark space shifts toward the anode. At sufficiently high values of $U_{d c}$, when the anode is located inside the negative glow, the anode glow disappears and the voltage drop across the anode sheath sharply decreases.

In our experiments, the value of the anode voltage drop was always positive. However, as was shown in [10], the anode voltage drop becomes negative at sufficiently low gas pressures. For the negative anode voltage drop to be obtained in our discharge tubes, a glow discharge should be presumably ignited in the pressure range $p<0.01$ torr at discharge voltages $U_{d c}>1000 \mathrm{~V}$ (however, this was beyond our experimental conditions).

\section{CONCLUSIONS}

In this paper we have examined the initiation and characteristics of a glow discharge in air in large-diameter discharge tubes. The main results can be summarized as follows:

(i) As the interelectrode distance $L$ increases, the breakdown curves $U_{d c}(p L)$ shift toward higher values of $U_{d c}$ and $p L$; i.e., a deviation from the Paschen law is observed.

(ii) The range of $p L$ for the normal regime of a glow discharge is determined more exactly. It is shown that regimes with a normal current density are characterized not only by certain "normal" values of the cathode sheath parameters $\left(U_{c}=U_{n}, p d_{c}=p d_{n}, j / p^{2}=\left(j / p^{2}\right)_{n}\right)$, but also by the presence of ionization in the anode sheath. The normal discharge regime takes place at low air pressures only for $p L$ values such that the cathode sheath, negative glow, Faraday dark space, and anode sheath with the anode glow are present simultaneously in the discharge. These conditions refer only to $p L$ values lying to the right of the inflection point in the breakdown curve.

(iii) The cathode glow in the normal regime of an air discharge (for a duralumin cathode) is characterized by the following parameters: $U_{n}=280 \pm 2 \mathrm{~V}, p d_{n} \approx$ 0.3 torr $\mathrm{cm}$, and $\left(j / p^{2}\right)_{n} \approx 0.21 \mathrm{~mA} / \mathrm{cm}^{2}$ torr ${ }^{2}$.

(iv) The axial profiles of the ion density, plasma potential, and electron temperature, as well as the anode voltage drop, are measured at various air pressure values lying both to the left and to the right of the minimum of the breakdown curve. It is shown that, in the normal discharge regime, the anode voltage drop and electron temperature at the boundary of the anode sheath are sufficiently high for the electron-impact ionization of gas molecules in the anode sheath.

\section{REFERENCES}

1. G. Frencis, in Handbuch der Physik, Ed. by S. Flugge (Springer-Verlag, Berlin, 1956), Vol. 22, p. 53.

2. V. L. Granovskiŭ, Electric Current in a Gas: SteadyState Current (Nauka, Moscow, 1971).

3. Yu. P. Raĭzer, Teplofiz. Vys. Temp. 24, 984 (1986).

4. V. N. Melekhin and N. Yu. Naumov, Pis'ma Zh. Tekh. Fiz. 12, 99 (1986) [Sov. Tech. Phys. Lett. 12, 41 (1986)].

5. Yu. P. Raĭzer and S. T. Surzhikov, Pis'ma Zh. Tekh. Fiz. 13, 452 (1987) [Sov. Tech. Phys. Lett. 13, 186 (1987)].

6. Yu. P. Raizer, Gas Discharge Physics (Nauka, Moscow, 1987; Springer-Verlag, Berlin, 1991).

7. G. G. Lister, J. Phys. D 25, 649 (1992).

8. B. N. Klarfeld, L. G. Guseva, and A. S. PokrovskayaSoboleva, Zh. Tekh. Fiz. 36, 704 (1966) [Sov. Phys. Tech. Phys. 11, 520 (1966)].

9. B. N. Klarfeld, L. G. Guseva, and V. V. Vlasov, in Proceedings of the $X$ International Conference on Phenomena in Ionized Gases, Oxford, 1971, Contrib. Pap., Vol. 1, p. 97.

10. B. N. Klarfeld and N. A. Neretina, Zh. Tekh. Fiz. 30, 186 (1960) [Sov. Phys. Tech. Phys. 5, 169 (1960)].

11. Yu. B. Golubovskiı and Sh. Kh. al Hawat, Zh. Tekh. Fiz. 57, 44 (1987) [Sov. Phys. Tech. Phys. 32, 25 (1987)].

12. Yu. B. Golubovskiŭ, V. I. Kolobov, and Sh. Kh. al Hawat, Zh. Tekh. Fiz. 58, 1729 (1988) [Sov. Phys. Tech. Phys. 33, 1046 (1988)].

13. Yu. B. Golubovskiı̆, V. S. Nekuchaev, and N. S. Ponomarev, Zh. Tekh. Fiz. 68 (3), 25 (1998) [Sov. Phys. Tech. Phys. 43, 288 (1998)].

14. V. A. Nemchinskiŭ, Zh. Tekh. Fiz. 40, 416 (1970) [Sov. Phys. Tech. Phys. 15, 317 (1970)].

15. G. J. Schulz and S. C. Brown, Phys. Rev. 98, 1642 (1955).

16. O. V. Kozlov, Electric Probe in a Plasma (Atomizdat, Moscow, 1969).

17. N. A. Gorbunov, N. B. Kolokolov, and A. A. Kudryavtsev, Fiz. Plazmy 15, 1513 (1989) [Sov. J. Plasma Phys. 15, 881 (1989)].

18. J. M. Meek and J. D. Craggs, Electrical Breakdown of Gases (Clarendon, Oxford, 1953; Inostrannaya Literatura, Moscow, 1960).

19. L. Jacques, W. Bruynooghe, R. Boucique, and W. Wieme, J. Phys. D 19, 1731 (1986).

20. V. I. Kolobov and A. Fiala, Phys. Rev. E 50, 3018 (1994).

21. V. A. Lisovskiy and S. D. Yakovin, Zh. Tekh. Fiz. 70 (6), 58 (2000) [Tech. Phys. 45, 727 (2000)].

22. T. W. Dakin, J. Gerhold, Z. Krasucki, et al., in Proceedings of the International Conference on Large High Voltage Electric Systems, Paris, 1977, p. 1.

23. V. A. Lisovskiy and V. D. Yegorenkov, J. Phys. D 27, 2340 (1994).

24. V. A. Shver̆gert and I. V. Shver̆gert, Fiz. Plazmy 14, 347 (1988) [Sov. J. Plasma Phys. 14, 204 (1988)]. 
25. V. A. Shvergert and I. V. Shvergert, Fiz. Plazmy 15, 621 (1989) [Sov. J. Plasma Phys. 15, 363 (1989)].

26. Yu. P. Raĭzer and M. N. Shneřder, Fiz. Plazmy 15, 318 (1989) [Sov. J. Plasma Phys. 15, 184 (1989)].

27. T. J. Sommerer, W. N. G. Hitchon, and J. E. Lawler, Phys. Rev. A 39, 6356 (1989).

28. Yu. P. Ră̌zer and M. N. Shneřder, Teplofiz. Vys. Temp. 29, 1041 (1991).

29. V. I. Kolobov and L. D. Tsendin, Phys. Rev. A 46, 7837 (1992).

30. A. P. Korzhavyı̆ and V. I. Kristya, Zh. Tekh. Fiz. 63 (2), 200 (1993) [Tech. Phys. 38, 156 (1993)].

31. Yu. P. Raĭzer and M. N. Shneřder, Teplofiz. Vys. Temp. 35, 19 (1997).

32. J. M. Anderson, J. Appl. Phys. 31, 511 (1960).

33. G. S. Solntsev, A. I. Orlov, and V. A. Dovzhenko, Radiotekh. Élektron. (Moscow) 15, 1980 (1970).

34. L. Protin, G. Fleury, and C. Vautier, Vacuum 34, 791 (1984).

35. H. Sasaki, K. Nanbu, and M. Takahashi, Rep. Inst. Fluid Sci. 10, 259 (1997).
36. D. B. Graves and K. F. Jensen, IEEE Trans. Plasma Sci. 14, 78 (1986).

37. G. Lapenta, F. Iinoya, and J. U. Brackbill, IEEE Trans. Plasma Sci. 23, 769 (1995).

38. L. G. Christophorou and J. G. Carter, Chem. Phys. Lett. 2, 607 (1968).

39. J. J. Lowke and R. Morrow, IEEE Trans. Plasma Sci. 23, 661 (1995).

40. C. S. Lakshminarasimha and J. Lucas, J. Phys. D 10, 313 (1977).

41. J. J. Lowke, J. H. Parker, and C. A. Hall, Phys. Rev. A 15, 1237 (1977).

42. Yu. B. Golubovskiı̆, Sh. Kh. al Hawat, and L. D. Tsendin, Zh. Tekh. Fiz. 57, 1285 (1987) [Sov. Phys. Tech. Phys. 32, 760 (1987)].

43. V. A. Shver̆gert, Fiz. Plazmy 14, 1363 (1988) [Sov. J. Plasma Phys. 14, 801 (1988)].

Translated by N. Larionova 